\section{Forniceal Involvement in Wernicke Encephalopathy}

We read with special interest the article of Zuccoli et al ${ }^{1}$ entitled "MR Imaging Findings in 56 Patients with Wernicke Encephalopathy: Nonalcoholics May Differ from Alcoholics." The authors reported the imaging features of Wernicke encephalopathy (WE), which include symmetric alterations in the thalami, mammillary bodies, tectal plate, and periaqueductal area, and other less typical imaging findings. ${ }^{1,2}$ Atypical MR imaging features are represented by signal intensity abnormalities on cranial nerve nuclei and cerebellum dentate nuclei, vermis, red nuclei and caudate nuclei, splenium, cerebral cortex, and fornix. ${ }^{1,2}$ It is known that forniceal involvement is critical in memory impairment in a variety of diseases, including neoplastic, inflammatory, infectious, congenital, vascular, and metabolic conditions. ${ }^{3}$ We present an additional case of forniceal involvement in WE associated with severe cognitive disfunction.

An alcoholic 47-year-old man presented with acute mental confusion, severe gait ataxia, bilateral sixth-nerve paralysis, and gazeevoked nystagmus. Brain MR imaging showed symmetric hyperintensity on FLAIR (Fig 1A), T2-weighted (Fig 1B), and diffusionweighted images (Fig 1C) in the medial thalami, mammillary bodies, periaqueductal area, tectal plate, and floor of the fourth ventricle. Furthermore, the forniceal bodies were expanded and presented with high signal intensity on the same sequences. The diagnosis of WE was suggested on the basis of clinical presentation and MR imaging findings, and treatment with parenteral thiamine was started. After 2 weeks of therapy, the mental confusion was totally resolved, and follow-up brain MR imaging (Fig 1D) revealed improvement of most of the lesions. However, the patient persisted with profound loss of episodic memory. Recently, Zuccoli et $\mathrm{al}^{1}$ reported only 2 cases with forniceal involvement in a series of 56 patients with WE, both nonalcoholic. In addition, Sugai and Kikugawa ${ }^{4}$ related 1 case of alcoholic WE with involvement of the fornix. However, there was no mention of memory deficits in these patients.

We present this case of alcoholic WE demonstrating all the typical imaging findings and, in addition, the atypical and rare involvement of the fornix. As part of the hippocampal-diencephalic system, the fornices are thought to contribute to the efficient encoding and normal recall of new episodic information. ${ }^{3}$ Due to its critical role in the limbic system and in the function of memory, forniceal involvement should be routinely reviewed in cases in which memory impairment is a significant clinical feature. Several studies have demonstrated typical and atypical findings in WE, including forniceal abnormalities. ${ }^{1,2,4}$ Nevertheless, none of them correlated this alteration with cognitive deficits. We emphasize that because the fornix is easily overlooked at routine imaging, radiologists should be aware of the association between forniceal abnormalities and memory deficits and seek this diagnosis. Recognition of involvement of the fornix in WE could be helpful in prompt clinical assessment of cognitive function and in determining the risk of permanent memory deficits in these patients.

\section{References}

1. Zuccoli G, Santa Cruz D, Bertollini M, et al. MR imaging findings in 56 patients with Wernicke encephalopathy: nonalcoholics may differ from alcoholics. AJNR Am J Neuroradiol 2009;30:171-76. Epub 2008 Oct 22

2. Zuccoli G, Pipitone N. Neuroimaging findings in acute Wernicke's encephalopathy: review of the literature. AJR Am J Roentgenol 2009;192:501-08

3. Thomas AG, Koumellis P, Dineen RA. The fornix in health and disease: an imaging review. Radiographics 2011;31:1107-21

4. Sugai A, Kikugawa K. Atypical MRI findings of Wernicke encephalopathy in alcoholic patients. AJR Am J Roentgenol 2010;195:W372-73, author reply W374

R.S. Borges

N. Ventura

E.L. Gasparetto

Department of Radiology

M.V.R. Pinto

Department of Neurology

University Federal of Rio de Janeiro

Rio de Janeiro, Brazil

http://dx.doi.org/10.3174/ajnr.A2888
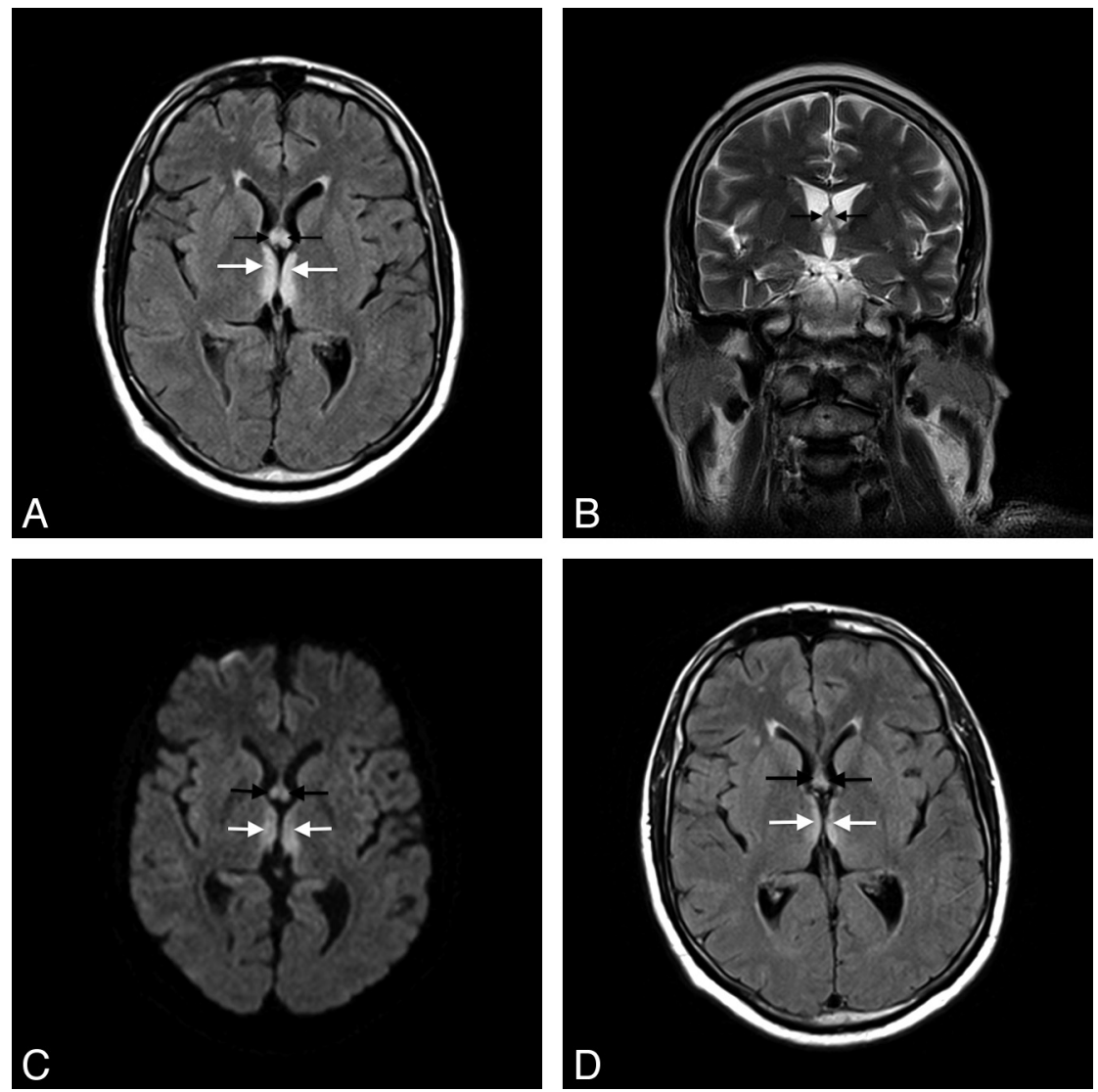

Fig 1. A, Axial FLAIR image shows high signal intensity symmetrically distributed in the medial thalami (white arrows) and fornices (black arrows). B, Coronal T2-weighted MR image shows increased size and signal intensity of the forniceal bodies (black arrows). C, Axial diffusion-weighted image shows high signal intensity in the columns of the fornix (black arrows) and medial thalami (white arrows). D, After 1 week of thiamine therapy, axial FLAIR image shows important reduction in the symmetric hyperintensity in the medial thalami (white arrows) and fornices (black arrows). 